\title{
Fourier transforms of convolution operators
}

\author{
by \\ CHARLES SWARTZ (Las Cruces, New Mexico)
}

\begin{abstract}
Let $\Phi \subseteq S$ be a test space and $\Psi$ the image of $\Phi$ under the Fourier transform. It is shown under certain conditions on $\Phi$ that the Fourier transform is an algebraic and topological isomorphism from the space of convolution operators on $\Phi$ onto the space of multipliers on $\Psi$.
\end{abstract}

A very important theorem of $\mathrm{L}$. Schwartz states that the Fourier transform is an algebraic and topological isomorphism from the space of convolution operators on $\mathscr{S}^{\prime}$ onto the space of multipliers on $\mathscr{S}^{\prime}$ (Theorem VII. XXV of [3]). The analogous result for the space of distributions of exponential order has been established by Zieleźny [9]. In this paper we establish a generalization of these results; our final result will yield the theorems of L. Schwartz and Zieleźny as special cases.

This paper is a continuation of [5], where convolution operators on. generalized function spaces are discussed. In Section 1 we consider multipliers on certain test spaces and supply the space of multipliers with a locally convex topology. Various properties of the topology are then discussed. In Section 2 the Fourier transform for certain generalized function spaces is discussed as in [1], and we show that under certain conditions the Fourier transform is an algebraic and topological isomorphism from the space of convolution operators onto the space of multipliers. In the final section we consider the results in Section 2 for certain test spaces. In particular the generalization of Schwartz's result is shown to hold for $K\left\{M_{p}\right\}$ spaces which satisfy conditions (M), (N), and (F) as set forth in [4], [5]. This class of spaces includes $\mathscr{S}$ and the test space $\mathscr{K}_{1}$ of functions of exponential growth as treated by Zieleźny [9].

Our terminology and notation will be as in [5].

1. The space of multipliers. Throughout this section $\Psi$ will denote an arbitrary vector space of infinitely differentiable functions on $R^{k}$ equipped with a locally convex topology such that differentiation is continuous on $\Psi$. We first define the multipliers on $\Psi$.

Definturon. The space of multipliers on $\Psi, \mathcal{O}_{M}(\Psi)$, is the set of all infinitely differentiable functions $\alpha$ on $R^{k}$ such that 
(i) $a \psi \epsilon \Psi$ for each $\psi \epsilon \Psi$,

(ii) the map $\psi \rightarrow \alpha \psi$ is continuous on $\Psi$.

If $\alpha \in \mathcal{O}_{M}(\Psi)$ and $T \epsilon \Psi^{\prime}$, the product of $\alpha$ and $T$ is defined by $\langle\alpha T, \psi\rangle$ $=\langle T, a \psi\rangle$ for $\psi \epsilon \Psi$. That is, the map $T \rightarrow a T$ is the adjoint of the map in (ii), and this mapping is therefore continuous with respect to either the weak* or strong topologies of $\Psi^{\prime}([6]$, II. 19. 5).

We note that $\mathcal{O}_{M}(\Psi)$ is closed under differentiation.

Propostrion 1. If $\alpha \in \mathcal{O}_{M}(\Psi)$, then $D^{j} a \in \mathcal{O}_{M}(\Psi)$ for $j \in N^{k}$, where $N$ denotes the set of non-negative integers.

Proof. For $\varphi \in \Psi$,

$$
{ }_{\varphi} D^{j} \alpha=\sum_{i \leqslant j}(-1)^{|i|}\left(\begin{array}{l}
j \\
i
\end{array}\right) D^{j-i}\left\{a D^{i} \varphi\right\} .
$$

The result now follows from (1) and the fact that differentiation is continuous on $\Psi$.

For the case $\Psi=\mathscr{S}$, the space of rapidly decreasing functions, the space $\mathcal{O}_{M}(\mathscr{S})$ is just the space $\mathcal{O}_{M}$ introduced by L. Schwartz ([3], VII. 5). (See [6], II. 25. 5 for the equivalence of the definition above and that of Schwartz.) The space $\mathcal{O}_{M}$ is supplied with a locally convex topology such that a sequence $\left\{a_{j}\right\} \subseteq \mathcal{O}_{M}$ converges to 0 iff for each $\varphi \in \mathscr{S}, \varphi D^{P} a_{j} \rightarrow 0$ such that a sequence $\left\{a_{j}\right\} \subseteq \mathcal{M}_{M}$ converge to 2.4.15 and [3], VII. 5). With respect to this locally convex topology the bilinear map $(\alpha, \varphi) \rightarrow a p$ from $\mathcal{O}_{M} \times \mathscr{S} \rightarrow \mathscr{S}$ is hypocontinuous with respect to the bounded subsets of $\mathscr{S}$.

Proposition 2. The sequence $\left\{\alpha_{j}\right\} \subseteq \mathcal{O}_{M}$ converges to 0 in $\mathcal{O}_{M}$ iff for each $\varphi \in \mathscr{S}, \alpha_{j} \varphi \rightarrow 0$ in. $\mathscr{S}$ uniformly for $\varphi$ varying over a bounded subset of $\mathscr{S}$.

Proof. Suppose $a_{j} \rightarrow 0$ in $\mathcal{O}_{M}$. From the hypocontinuity of the bilinear map $(\alpha, \varphi) \rightarrow \alpha \varphi$ from $\mathcal{O}_{M} \times \mathscr{S} \rightarrow \mathscr{S}, \alpha_{j} \varphi \rightarrow 0$ in $\mathscr{S}$ uniformly when $\varphi$ varies over a bounded subset of $\mathscr{S}$.

Let $B$ be a bounded subset of $\mathscr{S}$. Since $a_{j} \varphi \rightarrow 0$ in $\mathscr{S}$ uniformly for $\varphi \in B$, for each $i \in N^{k}, \varphi D^{i} \alpha_{j} \rightarrow 0$ in $\mathscr{S}$ uniformly for $\varphi \in B$ by (1). In particular, $\varphi D^{i} \alpha_{j} \rightarrow 0$ uniformly on $R^{k}$ for $\varphi \in B$ so that $\alpha_{j} \rightarrow 0$ in $\mathcal{O}_{M}$.

From Proposition 2 we obtain an interesting characterization of convergence in $\mathcal{O}_{c}^{\prime}([3]$, VII. 5).

CoROLLARY 3. A sequence $\left\{T_{n}\right\} \subseteq \mathcal{O}_{c}^{\prime}$ converges to 0 in $O_{c}^{\prime}$ iff for each $\varphi \in \mathscr{S}, T_{n} * \varphi \rightarrow 0$ in $\mathscr{S}$ uniformly when $\varphi$ varies over a bounded subset of $\mathscr{S}$.

Proof. That $T_{n} \rightarrow 0$ in $\mathcal{O}_{c}^{\prime}$ implies the condition stated follows from the fact that the bilinear map $(T, \varphi) \rightarrow T * \varphi$ from $\mathcal{O}_{c}^{\prime} \times \mathscr{S} \rightarrow \mathscr{S}$ is hypocontinuous.

Let $B \subseteq \mathscr{S}$ be bounded and suppose $T_{n} * \varphi \rightarrow 0$ in $\mathscr{S}$ uniformly for $\varphi \in B$. To show $T_{n} \rightarrow 0$ in $\mathcal{O}_{c}^{\prime}$ it suffices to show $\mathscr{F}\left\{T_{n}\right\} \rightarrow 0$ in $\mathcal{O}_{M}$, where
$\mathscr{F}\left\{T_{n}\right\}$ is the Fourier transform of $T_{n}$ ([3], VII. 8). For $\varphi \in B$, if $\psi=\mathscr{F}^{-1}\{\varphi\}$, $p \mathscr{F}\left\{T_{n}\right\}=\mathscr{F}\left\{T_{n} * \psi\right\} \rightarrow 0$ in $\mathscr{S}$ uniformly for $\varphi \in B$ since $\mathscr{F}^{-1}\{B\}$ is bounded in $\mathscr{S}$. Hence $\mathscr{F}\left\{T_{n}\right\} \rightarrow 0$ in $\mathcal{O}_{M}$ by Proposition 2.

Remark. This result shows that convergence in the topology introduced in [5] agrees with the convergence defined by L. Schwartz in [3], VII. 5 .

Motivated by Proposition 2 we introduce a locally convex Hausdorff topology on $\mathcal{O}_{M}(\Psi)$ by means of the semi-norms

$$
q_{p, B}(\alpha)=\sup \{p(\alpha \varphi): \varphi \epsilon B\},
$$

where $p$ is a continuous semi-norm on $\Psi$ and $B$ is a bounded subset of $\Psi$. We assume $\mathscr{O}_{M Y}(\Psi)$ is equipped with the locally convex topology generated by the semi-norms $q_{p, B}$, where $p$ runs over the family of continuous seminorms on $\Psi$ (or some generating family of semi-norms) and $B$ runs through the bounded subsets of $\Psi$. Thus, we have

Proposition 4. A net $\left\{a_{w}\right\}$ in $\mathcal{O}_{M}(\Psi)$ converges to 0 in $\mathcal{O}_{M}(\Psi)$ iff for each $\varphi \in \Psi, \alpha_{\nu} \varphi \rightarrow 0$ in $\Psi$, where the convergence is uniform for $\varphi$ varying in a bounded subset of $\Psi$.

Remark. Thus convergence in the topology defined on $\mathcal{O}_{M}(\mathscr{S})$ in this fashion agrees with the convergence defined by Schwartz in $\mathcal{O}_{M}$ (Proposition 2).

From Proposition 4, formula (1), and the fact that differentiation is continuous on $\Psi$, we also have

Propostinon 5. For any $j \in N^{k}$, the map $\alpha \rightarrow D^{j} \alpha$ is continuous on $\mathcal{O}_{M}(\Psi)$.

We summarize the continuity properties of the product map in the following statements.

Propostition 6. The bilinear map $(\alpha, \varphi) \rightarrow$ ap from $\quad \mathcal{O}_{M}(\Psi) \times \Psi \rightarrow \Psi$ is $\mathscr{B}$-hypocontinuous, where $\mathscr{B}$ is the family of bounded subsets of $\Psi$.

Proof. Fix $\alpha \in \mathcal{O}_{M}(\Psi)$. Then the map $\varphi \rightarrow a \varphi$ is continuous on $\Psi$ by definition of $\mathcal{O}_{M}(\Psi)$.

Let $B \subseteq \Psi$ be bounded and suppose the net $\left\{a_{y}\right\}$ converges to 0 in $\mathcal{O}_{M}(\Psi)$. Then by Proposition $4, a_{\nu} \varphi \rightarrow 0$ in $\Psi$, uniformly for $\varphi \in B$, and hence the result.

The dual of $\Psi$ equipped with the strong topology will be denoted by $\Psi_{b}^{\prime}$.

Proposition 7. The bilinear map $(\alpha, T) \rightarrow a$ from $\mathcal{O}_{M}(\Psi) \times \Psi_{b}^{\prime} \rightarrow \Psi_{b}^{\prime}$ is separately continuous.

Proof. Fix $a \in \mathcal{O}_{M}(\Psi)$. The map $T \rightarrow a T$ is the conjugate of the map defined in (ii), and is therefore continuous with respect to the strong otpologies ([6], II. 19.5). 
Fix $T \in \Psi^{\prime}$. Suppose the net $\left\{\alpha_{\nu}\right\}$ converges to 0 in $\mathcal{O}_{M}(\Psi)$. Let $B$ be bounded in $\Psi$. By Proposition $4, \alpha_{\nu} \varphi \rightarrow 0$ in $\Psi$ uniformly for $p \in B$ so $\left\langle T, \alpha_{\nu} \varphi\right\rangle=\left\langle\alpha_{v} T, \varphi\right\rangle \rightarrow 0$ uniformly for $\varphi \in B$. That is, $\alpha_{\nu} T \rightarrow 0$ in $\Psi_{b}^{\prime}$. Proposition 8. The bilinear map $(\alpha, \beta) \rightarrow \alpha \beta$ from $\mathcal{O}_{M}(\Psi) \times \mathcal{O}_{M}(\Psi) \rightarrow$ $\rightarrow \mathcal{O}_{M}(\Psi)$ is $\mathscr{B}$-hypocontinuous, where $\mathscr{B}$ is the family of all bounded subsets of $\mathcal{O}_{M}(\Psi)$.

Proof. Let $B$ be a bounded subset of $\mathcal{O}_{M}(\Psi)$ and let $\left\{\alpha_{\nu}\right\}$ be a net which converges to 0 in $\mathcal{O}_{M}(\Psi)$. If $A \subseteq \Psi$ is bounded, then $B A$ $=\{\alpha \varphi: \alpha \in B, \varphi \in A\}$ is bounded in $\Psi$ by Proposition 6 and Proposition 4.7.2 of [2]. Hence $\alpha_{\nu} \beta \rightarrow 0$ in $\mathcal{O}_{M}(\Psi)$, uniformly for $\beta \in B$ by Proposition 4.

We may also view multiplication by a fixed element of $\mathcal{O}_{M}(\Psi)$ as a linear operator on $\Psi$. That is, if $\alpha \in \mathcal{O}_{M}(\Psi), \alpha$ induces a continuous linear map $\alpha^{\#}: \Psi \rightarrow \Psi$ via $\alpha^{\#}: \varphi \rightarrow$ a . If we denote the space of all linear maps from $\Psi$ into $\Psi$ by $L(\Psi, \Psi)$, the map $a \rightarrow \alpha^{\#}$ imbeds $\mathcal{O}_{M}(\Psi)$ in $I(\Psi, \Psi)$. We denote by $\tau$ the topology induced on $\mathcal{O}_{M}(\Psi)$ by $L_{b}(\Psi, \Psi)$, where $L_{b}(\Psi, \Psi)$ is $L(\Psi, \Psi)$ equipped with the topology of uniform convergence on bounded subsets of $\Psi$. Proposition 4 can thus be rephrased as

Proposition 9. The topology $\tau$ coincides with the original topology of $\mathcal{O}_{M}(\Psi)$

As an application of this result, we show that certain spaces of multipliers are nuclear.

Proposimion 10. If $\Psi$ is complete and barreled, and $\Psi_{b}^{\prime}$ is nuclear and complete, then $\mathcal{O}_{M}(\Psi)$ is nuclear.

Proof. By Theorem III. 50.5 of [6], $L_{b}(\Psi, \Psi)$ is nuclear, and hence $\mathcal{O}_{M}(\Psi)$ must be nuclear, being a subspace of a nuclear space.

This technique can also be used to show that $\mathcal{O}_{M}(\Psi)$ is complete for certain $\Psi$

Propostiron 11. Let the space $\Psi$ be such that the injection of $\Psi$ into $\mathscr{E}$ is continuous and for every compact subset $K$ of $R^{t h}$ there is a $\varphi \in \Psi$ such that $\varphi(t) \neq 0$ for $t \in K$. If $\Psi$ is complete and bornological, then $\mathcal{O}_{M}(\Psi)$ is complete.

Proof. Let $\left\{\alpha_{v}\right\}$ be a Cauchy net in $\mathcal{O}_{M}(\Psi)$. Then for each $x \in R^{k}$, $\left\{a_{v}(x)\right\}$ converges; for if $\varphi \in \Psi$ is such that $\varphi(x)=1$, then $\left\{\alpha_{\nu} \varphi\right\}$ is a Cauchy net in $\Psi$, and therefore is a Cauchy net in $\mathscr{E}$, and thus $\left\{\alpha_{\nu}(x) p(x)\right\}=\left\{\alpha_{\nu}(x)\right\}$ converges. Set $\alpha(x)=\lim \alpha_{\nu}(x)$.

Now $\left\{\alpha_{v}^{\#}\right\}$ is Cauchy in $L_{b}(\Psi, \Psi)$, and therefore there exists $T \in L_{b}(\Psi, \Psi)$ such that $a_{v}^{\#} \rightarrow T$ in $L_{b}(\Psi, \Psi)$ ([6], Cor. 1 of II. 32.2). Moreover, from above we must have $T_{\varphi}=a p$ for each $p \in \Psi$.

It only remains to show that $\alpha \in \mathscr{E}$, for then $T=\alpha^{\#}$ and $\alpha_{p} \rightarrow \alpha$ in $\mathcal{O}_{M}(\Psi)$ by Proposition 9. If $K$ is a compact subset of $R^{k}$ and $\varphi \in \Psi$ is such that $\varphi(t) \neq 0$ for $t \epsilon K$, then $\alpha_{\nu} \varphi \rightarrow \alpha \varphi$ in $\Psi$, and therefore $\alpha_{\nu} \varphi \rightarrow \alpha \varphi$ in $\mathscr{E}$, and hence $\alpha_{\nu}(t) \rightarrow \alpha(t)$ uniformly for $t \in \mathbb{K}$ since $\varphi$ is bounded away from 0 on $K$. But, $\left\{D^{j} \alpha_{p}\right\}$ is also Cauchy in $\mathcal{O}_{M}(\Psi)$ for any $j$ (Proposition 5) so by the argument above $\left\{D^{j} \alpha_{\nu}\right\}$ converges uniformly on compact subsets of $R^{k}$. Thus $\alpha$ is infinitely differentiable.

It is clear that this result is applicable to a wide class of test spaces For example, it holds for the spaces $\mathscr{D}$ and $\mathscr{S}$, the space $Z$ of Gelfand and Shilov [1], and the space $\mathscr{K}_{1}$ of Zieleźny [9].

In a similar fashion, if $\alpha \in \mathcal{O}_{M}(\Psi)$, then $\alpha$ induces a continuous linear $\operatorname{map} \alpha^{*}: \Psi_{b}^{\prime} \rightarrow \Psi_{b}^{\prime}$ by $\alpha^{*}(T)=\alpha T$. The map $\alpha \rightarrow \alpha^{*}$ imbeds $\mathcal{O}_{M}(\Psi)$ in $L\left(\Psi_{b}^{\prime}, \Psi_{b}^{\prime}\right)$. We denote by $L_{\varepsilon}\left(\Psi_{b}^{\prime}, \Psi_{b}^{\prime}\right)$ the space $L\left(\Psi_{b}^{\prime}, \Psi_{b}^{\prime}\right)$ equipped with the topology of uniform convergence on equicontinuous subsets of $\Psi_{b}^{\prime}$ If $\tau^{\prime}$ denotes the topology induced on $\mathcal{O}_{M}(\Psi)$ by $L_{\varepsilon}\left(\Psi_{b}^{\prime}, \Psi_{b}^{\prime}\right)$, we have

Propostrmon 12. The topology $\tau^{\prime}$ coincides with the original topology defined on $\mathcal{O}_{M}(\Psi)$.

Proof. Let $\left\{\alpha_{\nu}\right\}$ be a net in $\mathcal{O}_{M}(\Psi), A$ be an equicontinuous subset of $\Psi_{b}^{\prime}$, and $B$ be a bounded subset of $\Psi$. Then $\alpha_{v}^{*} \rightarrow 0$ in $\tau^{\prime}$ iff $\alpha_{v}^{*}(T) \rightarrow 0$ in $\Psi_{b}^{\prime}$ uniformly for $T \in A$ iff $\left\langle\alpha_{\nu} T, \varphi\right\rangle \rightarrow 0$ uniformly for $T \in A, \varphi \in B$ iff $\alpha_{v} \varphi \rightarrow 0$ in $\Psi$ uniformly for $\varphi \in B([2], 3.4 .7)$ iff $\alpha_{\nu} \rightarrow 0$ in $\mathcal{O}_{M}(\Psi)$.

Remark. See the remark following Theorem VII. X in [3] for a similar statement pertaining to $\mathcal{O}_{M}(\mathscr{S})$,

2. The Fourier transform. Let $\Phi$ be a test space (see [5] for the definition) with a continuous translation such that $\Phi \subseteq \mathscr{S}$ with the injection continuous. If $f$ denotes a function in $L^{1}\left(R^{k}\right)$, we denote by $\mathscr{F}\{f\}$ its Fourier transform, $\mathscr{F}\{f\}(x)=\int e^{i(x, t)} f(t) d t$, and we set $\Psi=\{\mathscr{F}\{\varphi\}: \varphi \epsilon \Phi\}$. (In the terminology of [1], III.1.3, the space $\Psi$ is called the dual space with respect to $\Phi$.) The vector spaces $\Phi$ and $\Psi$ are algebraically isomorohic via the Fourier transform, and we assume that the topology of $\Psi$ is that inherited from $\Phi$ by means of this isomorphism. If $T \epsilon \Phi^{\prime}$, its Fourier transform, $\mathscr{F}\{T\}$, is the element of $\Psi^{\prime}$ defined by $\langle\mathscr{F}\{T\}, \mathscr{F}\{\varphi\}\rangle$ $=(2 \pi)^{k}\langle T, \varphi\rangle$ ([1], III.2.1). Thus $\mathscr{F}$ is a linear operator from $\Phi^{\prime}$ into $\Psi^{\prime}$, and since $\mathscr{F}$ is essentially the adjoint of the inverse Fourier transform from $\Psi$ into $\Phi, \mathscr{F}$ is continuous with respect to the strong (or weak ${ }^{*}$ ) topologies of these spaces ([6], II.19.5).

Throughout this section we make the assumption that the test space $\Phi$ is such that any polynomial is a multiplier on $\Phi$. Then $\Phi$ is closed under differentiation and multiplication by polynomials and the formulas

$$
P(D) \mathscr{F}\{T\}=\mathscr{F}\{P(i x) T\}, \quad \mathscr{F}\{P(D) T\}=P(-i \sigma) \mathscr{F}\{T\}
$$

hold for $T \epsilon \Phi^{\prime}$ and $P$ a polynomial ([1], III.2.1). From (3) it also follows that multiplication by polynomials and differentiation are continuous operators on $\Psi$. Hence the space $\Psi$ satisfies the conditions set forth in Section 1 for multipliers. 
In this section we generalize Theorem VII. XXV of [3] which states that $\mathcal{O}_{c}^{\prime}$ and $\mathcal{O}_{M}$ are topologically isomorphic under the Fourier transform. We show that for certain test spaces $\mathcal{O}_{M}(\Psi)$ and $\mathcal{O}_{c}^{\prime}(\Phi)$ are topologically isomorphic under the Fourier transform as defined above. of $[3]$.

We first establish a simple version of the exchange formula VII.8.5

LEMIMA 13. $\mathcal{O}_{c}^{\prime}(\Phi) \subseteq \mathcal{O}_{c}^{\prime}(\mathscr{S})$

Proof. Let $T \in \mathcal{O}_{c}^{\prime}(\Phi)$. If $p \in \mathscr{D}$, then $\varphi \in \Phi$ so that $T * p \in \Phi \subseteq \mathscr{S}$. Thus $T \in \mathscr{O}_{c}^{\prime}(\mathscr{S})$ by the remarks in [3], VII.5.

LEMTNA 14. If each $p \epsilon \Phi$ is a convolution operator on $\Phi$, i.e., $\phi \in \mathcal{O}_{c}^{\prime}(\Phi)$, then $\mathscr{F}\{\varphi\}$ is a multiplier on $\Psi$.

Proof. Suppose $\psi_{1}=\mathscr{F}\left\{\varphi_{1}\right\} \in \Psi$ with $\varphi_{1} \in \Phi$. Then

$$
\mathscr{F}\{\varphi\} \psi_{1}=\mathscr{F}\left\{\varphi * \varphi_{1}\right\} \in \Psi .
$$

Also if $\left\{\varphi_{\nu}\right\}$ is a net in $\Phi$ which converges to 0 , then (4) shows that $\mathscr{F}\{\varphi\} \mathscr{F}\left\{\varphi_{v}\right\} \rightarrow 0$ in $\Psi$. Hence $\mathscr{F}\{\varphi\}$ belongs to $\mathcal{O}_{M}(\Psi)$.

TheOREM 15. If each $\varphi \in \Phi$ is in $\mathcal{O}_{c}^{\prime}(\Phi)$, then for each $T \in \mathcal{O}_{c}^{\prime}(\Phi)$

$$
\mathscr{F}\{\varphi * T\}=\mathscr{F}\{\varphi\} \mathscr{F}\{T\} \text {. }
$$

Proof. By Lemma $13, T \in \mathcal{O}_{c}^{\prime}(\mathscr{S})$ so that $\mathscr{F}\{T\}$ is an infinitely differentiable function (see the first part of the proof of Theorem II.30.3 of [6] where this fact is established). Therefore the elements on the right and. left side of (5) are both infinitely differentiable functions, and to show that they are equal it suffices to show that they are equal as elements of $Z^{\prime}$ ([1], III.1.2). For $\alpha \in \mathscr{D}$, by hypothesis, we have

$$
\langle\mathscr{F}\{\varphi * T\}, \mathscr{F}\{\alpha\}\rangle=(2 \pi)^{k}\langle\varphi * T, \alpha\rangle=(2 \pi)^{k}\langle T, \varphi * \alpha\rangle .
$$

But by Lemma 14,

$$
\langle\mathscr{F}\{\varphi\} \mathscr{F}\{T\}, \mathscr{F}\{\alpha\}\rangle=\langle\mathscr{F}\{T\}, \mathscr{F}\{\varphi * \alpha\}\rangle=(2 \pi)^{k}\langle T, \varphi * \alpha\rangle,
$$

and the equality follows.

Formula (5) is the eritical point in much of the discussion that follows. In the final section we consider test spaces for which this formula holds. Note that we have not used the full statement of Theorem VIr. XXV of [3], but only the fact that if $T \in \mathcal{O}_{c}^{\prime}$, then $\mathscr{F}\{T\}$ is an infinitely differentiable function.

THEOREM 16. If for each $p \in \Phi$ and each $T \in \mathcal{O}_{c}^{\prime}(\Phi), \mathscr{F}\{T * \varphi\}=\mathscr{F}\{T\} \mathscr{F}\{p\}$, then $\mathscr{F}\left\{\mathcal{O}_{c}^{\prime}(\Phi)\right\} \subseteq \mathcal{O}_{M}(\Psi)$.

Proof. Let $T \in \mathcal{O}_{c}^{\prime}(\Phi)$ and again note that $\mathscr{F}\{T\}$ is an infinitely differentiable function. If $\psi=\mathscr{F}\{\varphi\} \in \Psi$ with $\varphi \in \Phi$, then $\psi \mathscr{F}\{T\}$
$=\mathscr{F}\{T * \varphi\} \epsilon \Psi$. Also if $\left\{\varphi_{\psi}\right\}$ is a net in $\Phi$ which converges to 0 in $\Phi$, then $\mathscr{F}\left\{\varphi_{v}\right\} \mathscr{F}\{T\}=\mathscr{F}\left\{T * \varphi_{\nu}\right\} \rightarrow 0$ in $\Psi$. Hence $\mathscr{F}\{T\}$ is a multiplier on $\Psi$. THEOREM 17. If for each $\varphi \epsilon \Phi$ and each $T \in \mathcal{O}_{c}^{\prime}(\Phi), \mathscr{F}\{T * \varphi\}=\mathscr{F}\{T\} \mathscr{F}\{\varphi\}$, then $\mathscr{F}: \mathcal{O}_{c}^{\prime}(\Phi) \rightarrow \mathcal{O}_{M}(\Psi)$ is continuous.

Proof. Let $\left\{T_{v}\right\}$ be a net in $\mathcal{O}_{c}^{\prime}(\Phi)$ which converges to 0 and let $B \subseteq \Psi$ be bounded. Then $A=\mathscr{F}^{-1}\{B\}$ is bounded in $\Phi$ so that $T_{\nu} * \varphi \rightarrow 0$ in $\Phi$ uniformly for $\varphi \in A$. Hence $\mathscr{F}\left\{T_{v} * \varphi\right\}=\mathscr{F}\left\{T_{v}\right\} \mathscr{F}\{\varphi\} \rightarrow 0$ in $\Psi$ uniformly for $\mathscr{F}\{\varphi\} \in B$, and, therefore, $\mathscr{F}\left\{T_{v}\right\} \rightarrow 0$ in $\mathcal{O}_{M}(\Psi)$.

From Theorems 16 and 17 we see that the Fourier transform is a linear continuous map from $\mathcal{O}_{c}^{\prime}(\Phi)$ into $\mathcal{O}_{M}(\Psi)$ provided that formula (5) is valid, and Theorem 15 gives a sufficient condition for this formula to be satisfied. To establish the analogue of Theorem VII. XXV of [3], we now show that $\mathscr{F}$ carries $\mathcal{O}_{c}^{\prime}(\Phi)$ onto $\mathcal{O}_{M}(\Psi)$. This fact is essentially established in Theorem 1 of [1], III.3.7. To apply this result, we only need to make the following observation.

LEMMA 18. If the infinitely differentiable function $g$ is in $\mathcal{O}_{M}(\Psi)$, then $g \in \Psi^{\prime}$.

Proof. Since $\mathscr{D}$ is sequentially dense in $\mathscr{S}^{\prime}$, there is a sequence $\left\{\varphi_{n}\right\} \subseteq \mathscr{D}$ such that $\varphi_{n} \rightarrow \delta$ in $\mathscr{S}_{b}^{\prime}$. Then $\mathscr{F}\left\{\varphi_{n}\right\} \rightarrow 1$ in $\mathscr{S}_{b}^{\prime}$, and hence $\mathscr{F}\left\{p_{n}\right\} \rightarrow 1$ in $\Psi_{b}^{\prime}$ since the injection $\Phi \rightarrow \mathscr{S}$ is continuous. Therefore, $g \mathscr{F}\left\{\varphi_{n}\right\} \rightarrow g$ in $\Psi_{b}^{\prime}$ and $g \in \Psi^{\prime}$.

By the lemma if $g \in \mathscr{O}_{M}(\Psi)$, then $g$ has an inverse Fourier transform and Theorem 1 of [1], III.3.7 gives

THEOREM 19. If $g \in \mathcal{O}_{M}(\Psi)$, then $\varphi=\mathscr{F}^{-1}\{g\}$ is a convolution operator on $\Phi$. That is, $\mathscr{F}^{-1}\left\{\mathcal{O}_{M}(\Psi)\right\} \subseteq \mathcal{O}_{c}^{\prime}(\Phi)$.

We now establish our generalization of Schwartz's result.

THEOREM 20. Suppose the test space $\Phi$ is such that for each $\varphi \in \Phi$ and each $T \in \mathcal{O}_{c}^{\prime}(\Phi), \mathscr{F}\{T * \varphi\}=\mathscr{F}\{T\} \mathscr{F}\{\varphi\}$. Then the Fourier transform is an algebraic and topological isomorphism from $\mathcal{O}_{c}^{\prime}(\Phi)$ onto $\mathcal{O}_{M}(\Psi)$.

Proof. From Theorems 17, 18, and 19, the Fourier transform is a continuous isomorphism from $\mathcal{O}_{c}^{\prime}(\Phi)$ onto $\mathcal{O}_{M}(\Psi)$. It remains only to show $\mathscr{F}^{-1}$ is continuous. Suppose $\left\{\psi_{v}\right\}$ is a net in $\mathcal{O}_{M}(\Psi)$ which converges to 0 and $\mathscr{F}\left\{T_{v}\right\}=\psi_{v}$ with $T_{v} \in \mathcal{O}_{c}^{\prime}(\Phi)$. Let $A \subseteq \Phi$ be bounded so that $B=\mathscr{F}\{A\}$ is bounded in $\Psi$. Then $\mathscr{F}\{\varphi\} \psi_{\nu}=\mathscr{F}\left\{T_{v} * \varphi\right\} \rightarrow 0$ in $\Psi$ uniformly for $p \in A$, and therefore $T_{\nu} * p \rightarrow 0$ in $\Phi$ uniformly for $p \in A$. That is, $T_{v} \rightarrow 0$ in $\mathcal{O}_{c}^{\prime}(\Phi)$.

Again we remark that the critical point in the analysis above is the validity of formula (5). Under the assumption that (5) holds it is easy to establish the familiar exchange formula $\mathscr{F}\{T * S\}=\mathscr{F}\{T\} \mathscr{F}\{S\}$, where $T \in \mathcal{O}_{c}^{\prime}(\Phi)$ and $S \in \Phi^{\prime}$.

Next we consider Theorem 20 for some of the classical test spaces. 


\section{Specific test spaces.}

EXAMPLE 1. If we take for the test space $\Phi$ the space $\mathscr{D}$ of L. Schwartz, then $\mathscr{O}_{c}^{\prime}(\mathscr{D})=\mathscr{E}^{\prime}$, and $\Psi=Z$ ([1], III.1.2). Any polynomial is certainly a multiplier on $\mathscr{D}$ and condition (5) is clearly satisfied in this case so Theorem 20 is applicable, and the Fourier transform is an algebraic and topological isomorphism from $\mathscr{E}^{\prime}$ onto $\mathcal{O}_{M}(Z)$. The class of multipliers on $Z$ is given in [1], II.3.2; a function $g$ is in $\mathcal{O}_{M}(Z)$ iff $g$ is entire and there are constants $b>0, \dot{m} \geqslant 0$, and $M>0$ such that $|g(z)| \leqslant M\left(1+|z|^{m}\right)$ $\exp (b|y|)$, where $z=x+i y$. Thus the fact that $\mathscr{F}$ carries $\mathscr{E}^{\prime}$ onto $\mathcal{O}_{M}(Z)$ is just the Paley-Wiener-Schwartz Theorem ([8], VI.4).

The other test spaces which we consider are the $K\left\{M_{p}\right\}$ spaces introduced by Gelfand and Shilov [1]. Further we restrict attention to those $K\left\{M_{p}\right\}$ spaces satisfying conditions (M), (N), and (F) as set forth in [1], [4], [5]. Our first result shows that formula (5) of Theorem 15 is valid for these test spaces.

THEOREM. 21. Let $\Phi=K\left\{M_{p}\right\}$, where the sequence satisfies conditions $(\mathrm{M}),(\mathrm{N})$, and $(\mathrm{F})$. Then the bitinear map $(p, \psi) \rightarrow{ }^{*} \psi$ is continuous from $\varphi \times \Phi \rightarrow \Phi$. hen

Proof. Let $p$ be a positive integer and $|j| \leqslant p, j \in N^{*}$. If $\varphi, \psi \in \Phi$,

$$
\begin{aligned}
\int M_{p}(x)\left|D^{j} \varphi^{*} \psi(x)\right| d x & =\int|\varphi(y)| \int M_{p}(x)\left|D^{j} \psi(x-y)\right| d x d y \\
& \leqslant C_{q} \int M_{q}(y)|\varphi(y)| d y \int M_{q}(t)\left|D^{j} \psi(t)\right| d t \\
& \leqslant C_{q}\|\varphi\|_{q}^{\prime}\|\psi\|_{\alpha}^{\prime}
\end{aligned}
$$

where $p^{\prime}=q$ and $C_{q}$ are given by condition (F) ([4], [5]) and $\|\varphi\|_{q}^{\prime}$ $=\sup \left\{\int M_{q}\left|D^{j} \varphi\right|:|j| \leqslant q\right\}$. From (6) it follows that $\left\|\varphi^{*} \psi\right\|_{p}^{\prime} \leqslant C_{q}\|\varphi\|_{q}^{\prime}\|\psi\|_{q}^{\prime}$, and since the sequence of semi-norms $\left\{\|\|_{p}^{\prime}\right\}$ generates the topology of $\Phi$ ([1], II.4.2), the theorem is established.

CoRollary 22. If $\varphi \in \Phi$, then $\varphi \in \mathcal{O}_{c}^{\prime}(\Phi)$.

Thus if $\Phi=K\left\{M_{p}\right\}$ is such that $\Phi \subseteq \mathscr{S}$ with the injection continuous and if polynomials are multipliers on $\Phi$, then the Fourier transform is an algebraic and topological isomorphism from $\mathcal{O}_{c}^{\prime}(\Phi)$ onto $\mathcal{O}_{M}(\Psi)$ by Theorem 20.

EXAMPLE 2. If $M_{p}(x)=\left(1+|x|^{2}\right)^{P}$, then $K\left\{M_{p}\right\}=\mathscr{S}$ and the remarls above give exactly Theorem VII. XXV of [3] (see also [7]). (It is observed in [4] that $\left\{M_{p}\right\}$ satisfies conditions (M), (N), and (F).) Note that the only fact used pertaining to the Fourier transform defined on $\mathcal{O}^{\prime}=O^{\prime}(\mathscr{S})$ was that $\mathscr{F}\{T\}$ is an infinitely differentiansform defined on $\mathscr{O}_{c}^{\prime}=\mathscr{O}_{c}^{\prime}(\mathscr{P})$

EXAMPLE 3. For $\left.M_{p}(x)=\exp (p \gamma(x)), \gamma(x)=(1+\infty)^{2}\right)^{1 / 2} \boldsymbol{O}_{c}$ EXAMPLE 3. For $M_{p}(x)=\exp (p \gamma(x)), \quad \gamma(x)=\left(1+|x|^{2}\right)^{1 / 2}, \mathbb{R}\left\{M_{p}\right\}$
$=\mathscr{K}_{1}([9])$ and $\left\{M_{p}\right\}$ satisfies conditions clear that $\mathscr{K}_{1} \subseteq \mathscr{S}$ with continuous injection and that polynomials are multipliers on $\mathscr{K}_{1}$. The remarks following Corollary 22 then give that the Fourier transform is an algebraic and topological isomorphism from $\mathcal{O}_{c}^{\prime}\left(\mathscr{K}_{1}\right)$ onto $\mathcal{O}_{M}\left(\mathscr{F}\left\{\mathscr{K}_{1}\right\}\right)$. This fact is just Theorem 3 of [9].

Theorem 20 may also be applied to inductive limits of $K\left\{M_{p}\right\}$ spaces like those treated in Theorem 21. This follows from the following observation.

Proposimion 23. Let $\Phi$ and $\Phi_{n}(n=1,2, \ldots)$ be test spaces with continuous translations such that $\Phi_{n} \subseteq \Phi_{n+1}$ and $\Phi=\bigcup_{1}^{\infty} \Phi_{n}$. Suppose the injection of $\Phi_{n}$ into $\Phi_{n+1}$ is continuous and $\Phi=\operatorname{ind} \Phi_{n}$. If $\Phi_{n} \subseteq \mathcal{O}_{c}^{\prime}\left(\Phi_{n}\right)$ for each $n$, then $\Phi \subseteq \mathcal{O}_{c}^{\prime}(\Phi)$.

Proof. Let $\varphi \in \Phi$. Then $\varphi \in \Phi_{N}$ for some $N$. The continuity of the map $\psi \rightarrow \varphi^{*} \psi$ from $\Phi$ into $\Phi$ follows from the fact that for $n \geqslant N$ the map $\psi \rightarrow \varphi^{*} \psi$ is continuous from $\Phi_{n}$ into $\Phi_{n}$ ([6], III.50).

Thus Theorem 20 is applicable to the $\mathscr{S}$-spaces of Gelfand and Shilor

\section{References}

[1] I. Gelfand and G. Shilov, Generalized Functions II, N. Y. 1968.

[2] J, Horvath, Topological Vector Spaces and Distributions, 1966.

[3] L. Schwartz, Théorie des distributions, Paris 1966.

[4] C. Swartz, Oonvolution in $K\left[M_{p}\right]$ spaces, Rocky Mountain Math. J., to appear.

[5] - Convergence of convolution operators, Studia Math. 42 (I972), pp. 249-257.

[6] F. Treves, Topological Vector Spaces, Distributions, and Kernels, N. Y. 1967.

[7] K. Yoshinaga, On the space $\left(\mathscr{S}^{\prime}\right)$ and the Fourier transform, Bull. Kyushu K. Yoshinaga, On the space
Inst. Tech., 5 (1959), pp. 1-11.

[8] K. Yosida, Functional Analysis, N. Y. 1965. [9] Z. Zieleźny, On the space of convolution operators in $\mathscr{K}_{1}^{\prime}$, Studia Math. 31 (1968),
pp. 111-124. 